\title{
Thrombotic Microangiopathy Triggered by COVID-19: Case Reports
}

\author{
Julia Korotchaeva ${ }^{a}$ Natalia Chebotareva ${ }^{a}$ Elena Andreeva ${ }^{a}$ Yuri Sorokin ${ }^{a}$ \\ Valerie McDonnell $^{\mathrm{a}}$ Ekaterina Stolyarevich ${ }^{\mathrm{b}}$ Sergey Moiseev ${ }^{\mathrm{a}}$ \\ aNephrology Sechenov First Moscow State Medical University, Moscow, Russia; ${ }^{b}$ Morphology Department, \\ Evdokimov Moscow State University of Medicine and Dentistry, Moscow, Russia
}

\section{Keywords}

Thrombotic microangiopathy · COVID-19 · Atypical

hemolytic uremic syndrome

\begin{abstract}
SARS-CoV-2 causes thrombotic microangiopathy (TMA) through the activation of an alternative and lectin complement pathway. TMA is one of the main reasons for acute kidney injury development in patients with COVID-19. In this study, we present 3 TMA cases with severe kidney injury triggered by SARSCoV-2. In the absence of other TMA causes, we diagnosed the atypical hemolytic uremic syndrome, triggered by SARS-CoV-2 due to abnormal complement activation. Because of both coagulation factors activation, and the high level of D-dimer in patients with COVID-19, it is crucial to differentiate disseminated intravascular coagulation from TMA. The use of anticomplement therapies such as eculizumab should be considered in refractory cases of progressive COVID-19. Controlled clinical trials are required before a definitive statement can be made.
\end{abstract}

(c) 2021 S. Karger AG, Basel

\section{Introduction}

Thrombotic microangiopathy (TMA) may be responsible for COVID-19 progression because of both direct endothelium damage by the virus along with severe inflammatory syndrome and coagulation cascades activation representing a marked and prolonged complement activation even in cases without the complement-system gene impairment. The mechanism of endothelial injury in patients with COVID-19 includes activation of the alternative and lectin complement pathway $[1,2]$. This suggestion was confirmed by the terminal complement complex C5b-9 deposits, C4d, and MASP2 detection in the pulmonary and skin capillaries in patients with severe COVID-19 [3]. Several cases were published on the matter of renal TMA, relapsing atypical hemolytic uremic syndrome (aHUS), and cortical necrosis associated with COVID-19 $[4,5]$. We report 3 cases of newly identified aHUS triggered by COVID-19.

\section{Case Presentations}

Case 1

We present a case of a 49-year-old female, who was admitted to the hospital complaining about abdominal pain, diarrhea, and vomiting. On admission, she was experiencing fever $39^{\circ} \mathrm{C}$, tachypnea, and normal blood pressure (BP). The PCR test for the detection of SARS-CoV-2 was positive. According to the CT scan results, the patient had $>50 \%$ lung involvement. Oxygen saturation was 95-96\%, so no respiratory support was required. Lab tests discovered anemia, mild thrombocytopenia, increased lactate dehydrogenase (LDH), and progressive loss in renal function. The patient developed oliguria due to acute kidney injury (AKI). Her peak creatinine was 558 $\mu \mathrm{mol} / \mathrm{L}$ on day 7 , which required hemodialysis (HD) (Table 1). karger@karger.com www.karger.com/nef (c) 2021 S. Karger AG, Base

Karger"
Correspondence to:

Natalia Chebotareva, natasha_tcheb@mail.ru 
Table 1. Laboratory parameters in case 1

\begin{tabular}{|c|c|c|c|c|c|c|}
\hline $\mathrm{BP}(\max ), \mathrm{mm} \mathrm{Hg}$ & & $130 / 80$ & $140 / 90$ & $150 / 90$ & $140 / 90$ & $130 / 85$ \\
\hline Hemoglobin, g/L & $120-150$ & 94 & 86 & 99 & 101 & 105 \\
\hline Thrombocytes $\times 10^{9} / \mathrm{L}$ & $180-320$ & 195 & 157 & 221 & 250 & 265 \\
\hline $\mathrm{LDH}, \mathrm{U} / \mathrm{L}$ & $<247$ & 580 & 515 & 491 & 169 & 160 \\
\hline & & & $\mathrm{HD}$ & HD & $\mathrm{HD}$ & \\
\hline International normalized ratio & $0.85-1.2$ & 1.02 & - & 1.15 & - & 0.99 \\
\hline PT, s & $11-16$ & 11.2 & - & 12.7 & - & 13.1 \\
\hline aPTT, s & $21.1-36.5$ & 24.7 & - & 26.3 & - & 28.2 \\
\hline Proteinuria, $\mathrm{g} / \mathrm{g} \mathrm{Cr}$ & $<0.15$ & 0.1 & 2.3 & 0.18 & 0.1 & 0.1 \\
\hline Hematuria in FOV & $0-1$ & $2-3$ & $2-3$ & $1-2$ & $1-2$ & $1-2$ \\
\hline
\end{tabular}

$\mathrm{LDH}$, lactate dehydrogenase; HD, hemodialysis.

Serology tests like ANCA, ANA, dsDNA, anti-GBM, and antiphospholipid antibody levels were unremarkable. ADAMTS 13 activity reached $69 \%$. The patient received treatment which included intravenous infusion of antibiotics, $40 \mathrm{mg}$ dexamethasone over 3 consequent days, and iron. The patient was discharged from the hospital with a negative PCR result for SARS-CoV-2 and a significant improvement in her lung function. However, the CRRT was still required.

Two months after COVID-19, the patient showed no renal function improvement. She was admitted to the nephrology and rheumatology clinic. The patient complained about impaired blurry vision. Her BP was moderately high (150/80-90 mm Hg), which was controlled by verapamil intake of $80 \mathrm{mg}$ /day. Retinopathy with reduced peripheral photosensitivity was subsequently confirmed. EGD confirmed the presence of erosive gastroduodenitis. A lab test demonstrated coombs-negative anemia, increased levels of $\mathrm{LDH}$, decreased levels of haptoglobin, and alternative complement pathway activation markers: decreased C3 levels 0.74 (normal range 0.82-1.85 g/L), normal C4 levels 0.3 (normal range 0.1-0.4), increased soluble C5b-9 levels, and normal platelet count (Table 1). A clinical urine test demonstrated mild proteinuria and no active urine sediment. The renal ultrasound was unremarkable. However, the Doppler ultrasound indicated a mosaic decrease in systolic blood flow velocity in segmental, interlobar, and arcuate arteries of both kidneys. A kidney biopsy was performed. The kidney sample contained 8 glomeruli. The glomeruli size was unremarkable, with no signs of mesangial and endocapillary hypercellularity. Four glomeruli demonstrated capillary loops ischemia with varying severity. The thickness of capillary loop walls was unremarkable. Interstitial sclerosis and tubular atrophy covered about $30 \%$ of the renal parenchyma. One medium/small artery showed signs of significant narrowing with hyperelastosis and intimal fibrosis due to arteriosclerosis. Arterioles showed signs of mucoid swelling. Immunofluorescence showed no trace of immunoglobulins, complement components (C3, C1q), kappa, lambda chains, and fibrin deposits. The diagnosis of TMA was confirmed based on lab findings as well as morphological changes: due to the presence of hemolytic anemia (low hemoglobin and haptoglobin levels, an increased LDH level, and the presence of schistocytes), decreased platelet count at COVID-19 manifestation, AKI stage 3, retinopathy, and gastrointestinal tract involvement. Other serology tests which included ANCA, ANA, and antiphospholipid antibodies were negative. Given the lack of evidence for thrombotic thrombocytopenic purpura, antiphospholipid syndrome, and other systemic diseases (systemic lupus erythematosus and ANCA-associated vasculitis), the atypical hemolytic uremic syndrome was diagnosed. The patient was offered eculizumab $900 \mathrm{mg}$ per week following 1,200 mg every 2 weeks. The treatment is yet being continued. Hemoglobin, $\mathrm{LDH}$, and haptoglobin levels were normalized (Table 1). Creatinine levels decreased after a month of eculizumab treatment (shown in Fig. 1a).

\section{Case 2}

We present a case of a 39-year-old male patient with a long history of mild hypertension. He was exposed to SARS-CoV-2 in late October 2020 and experienced fever up to $39^{\circ} \mathrm{C}$, weakness, dry cough, shortness of breath, and anosmia. According to CT scan results, the patient had viral bilateral polysegmental pneumonia with 50\% lung involvement.

After 10 days, he complained about the gradual progression of shortness of breath. The patient also experienced new symptoms such as nausea, vomiting, decreased diuresis, and visual impairment. His BP was $240 / 110 \mathrm{~mm} \mathrm{Hg}$. He was admitted to the hospital. Lab tests showed elevated levels of serum creatinine, low hemoglobin levels, and thrombocytopenia (Table 2). ADAMTS 13 activity was $71 \%$.

The urine test demonstrated mild proteinuria and erythrocyturia (Table 2). The patient received his treatment immediately. It included antibiotics, antiviral drugs, and oxygen therapy. Additionally, the patient received enoxaparin sodium 0.4 and prednisolone $500 \mathrm{mg}$ intravenous infusions. The patient also required HD. The patient's condition significantly improved over time. His temperature normalized, and the CT scan test showed positive dynamics (partial resolution of those early findings). However, some lab tests did not improve. The patient still showed signs of hemolysis, 
Fig. 1. Lab values in 3 cases before and after eculizumab therapy: case 1 (a); case 2 (b); and case $3(\mathbf{c})$. LDH, lactate dehydrogenase.

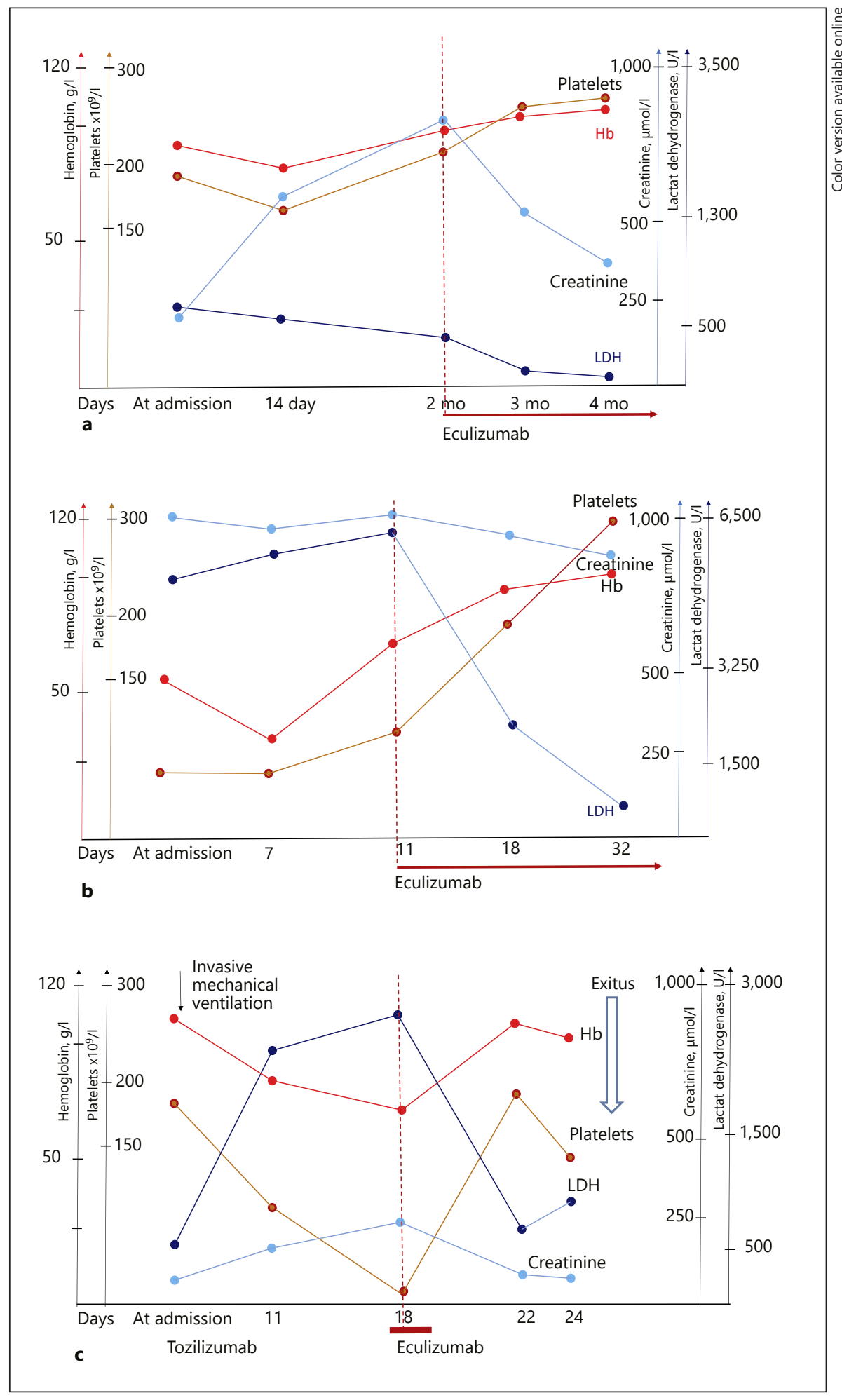

thrombocytopenia, and AKI (Table 2). Hence, plasma therapy was initiated but had to be interrupted shortly due to the development of pulmonary edema.
Systemic disease was excluded (no immunological signs of SLE, APS, and ANCA were found). Severe renal damage, platelet count over $30,000 / \mu \mathrm{L}$, and ADAMTS 13 activity allowed us to 
Table 2. Laboratory parameters in case 2

\begin{tabular}{lllllll}
\hline Parameters & $\begin{array}{l}\text { Normal } \\
\text { value }\end{array}$ & $\begin{array}{l}\text { At admission } \\
\text { (COVID-19) }\end{array}$ & $\begin{array}{l}\text { 7th day } \\
\text { (COVID-19) }\end{array}$ & $\begin{array}{l}\text { 11th day } \\
\text { eculizumab } \downarrow\end{array}$ & $\begin{array}{l}\text { 18th day } \\
\text { 32nd } \\
\text { day }\end{array}$ \\
\hline $\mathrm{BP}(\mathrm{max}), \mathrm{mm} \mathrm{Hg}$ & & $240 / 110$ & $200 / 110$ & $200 / 110$ & $160 / 100$ & $140 / 90$ \\
Oxygen saturation, \% & $>96$ & 97 & 98 & 96 & 98 & 99 \\
Hemoglobin, g/L & $120-150$ & 52 & 41 & 57 & 90 & 100 \\
Thrombocytes $\times 10^{9} / \mathrm{L}$ & $180-320$ & 54 & 60 & 51 & 198 & 297 \\
LDH, U/L & $<247$ & 5,300 & 5,645 & 6,200 & 2,100 & 600 \\
Creatinine, $\mu$ mol/L & $49-90$ & 1,035 & 1,010 & 1,030 & 975 & 900 \\
& & HD & HD & HD & HD & HD \\
International normalized ratio & $0.85-1.2$ & 1.1 & - & 1.0 & - & 1.0 \\
PT, s & $11-16$ & 13.2 & - & 13.0 & - & 14.1 \\
aPTT, s & $21.1-36.5$ & 24.6 & - & 24.2 & - & 25.1 \\
Proteinuria, g/g Cr & $<0.15$ & 0.2 & 0.1 & 0.2 & 0 & 0 \\
Hematuria, in FOV & $0-1$ & $5-6$ & $3-4$ & $0-1$ & $0-1$ & $0-1$ \\
\hline
\end{tabular}

$\mathrm{LDH}$, lactate dehydrogenase; BP, blood pressure; HD, hemodialysis.

exclude TTP. Hence, aHUS was diagnosed, which was probably triggered by COVID-19.

The patient was offered eculizumab. The patient was treated with 3 doses $900 \mathrm{mg}$ during 3 weeks. Soon after the treatment, the patient showed positive dynamics such as normalization of BP, hemoglobin and LDH levels, and the platelet count (shown in Fig. 1b). The patient's diuresis increased; however, the kidney function still did not fully recover. The patient refused further eculizumab treatment, so he remains on HD.

\section{Case 3}

We present a case of a 66-year-old female with a 10-year history of diabetes mellitus. She was exposed to SARS-CoV-2 in May 2020 and was admitted to the hospital with a fever up to $38.8^{\circ} \mathrm{C}$. According to CT scan results, the patient had viral pneumonia with $75 \%$ lung involvement. Oxygen saturation was $80-70 \%$. Lab tests demonstrated elevated CRP levels (249-413 mg/L), leukocytosis $\left(4.7 \times 10^{9} / \mathrm{L}\right), \mathrm{LDH}$ levels, decrease in $\mathrm{Hb}$ levels, platelets count, creatinine $(70 \mu \mathrm{mol} / \mathrm{L})$, fibrinogen $(12.77 \mathrm{~g} / \mathrm{L})$, and D-dimer $(2,700$ $\mathrm{ng} / \mathrm{mL}$ [normal values $<500 \mathrm{ng} / \mathrm{mL}]$ ). The patient developed acute respiratory distress syndrome and underwent invasive mechanical ventilation. The patient was offered tocilizumab $4 \mathrm{mg} / \mathrm{kg}$. After the short treatment, the patient showed positive dynamics. Her temperature normalized, and lab tests demonstrated the leukocyte number (4.7-6.8 $\left.\times 10^{9} / \mathrm{L}\right)$, CRP $(413-76 \mathrm{mg} / \mathrm{L})$, and fibrinogen (12.77-4.41 g/L). After a short-term improvement, the fever went back up to $38.9^{\circ} \mathrm{C}$, and lab tests demonstrated leukocytosis $(18 \times$ $\left.10^{9} / \mathrm{L}\right)$, an elevated CRP level ( $\left.186 \mathrm{mg} / \mathrm{L}\right)$, and a 20 -fold increase in procalcitonin. Enterococcus and Acinetobacter baumani were isolated from the sputum.

The patient also demonstrated symptoms of AKI: (oliguria and creatinine up to $193 \mu \mathrm{mol} / \mathrm{L}$ ), thrombocytopenia, anemia, increased LDH levels with 8-fold increase in the D-dimer level $(3,900 \mathrm{ng} / \mathrm{mL})$, and decreased fibrinogen level $(4.9 \mathrm{~g} / \mathrm{L}) \mathrm{com}-$ pared to that at admission. The patient received treatment that included meropenem, linezolid, cefoperazone-sulbactam, and colistin.
Disseminated intravascular coagulation (DIC) was the first reason explaining the development of microangiopathic syndrome in the patient with a severe infection. She was also offered fresh frozen plasma (FFP) transfusions. Her condition improved slightly after the treatment; her temperature normalized and lab tests stabilized, except for the CRP (13 mg/L), fibrinogen $(4.5 \mathrm{~g} / \mathrm{L})$, and $\mathrm{D}$-dimer $(2,000 \mathrm{ng} / \mathrm{mL})$. However, the patient still needed respiratory support (mechanical ventilation). She received antibiotics, platelet, and FFP transfusions $(750 \mathrm{~mL} /$ day). Prothrombin time was normal, and aPPT was slightly elevated (Table 3 ).

Despite all the treatment measures, the patient's microangiopathic syndrome kept progressing with severe thrombocytopenia, and the presence of schistocytes on a peripheral blood smear. ADAMTS 13 activity was 49\%. Other serology tests which included ANCA, ANA, and antiphospholipid antibodies were unremarkable.

The eculizumab infusion $900 \mathrm{mg}$ stabilized the platelet count, LDH levels, and creatinine levels (shown in Fig. 1c). However, the patient developed distal phalanges gangrene on her left hand with a generalized infection on the sixth day. The blood test showed the presence of Clostridium perfringens. Eculizumab transfusions were interrupted. FFP and LMWH transfusions were continued. Antibacterial therapy was changed to Zavicefta (ceftazidim/avibactam) and tigecycline. Despite all the taken measures, the patient died after 6 days.

\section{Discussion/Conclusion}

SARS-CoV-2 plays a pivotal role in complement activation and endothelial damage. Mannose-binding lectin binds to the SARS-CoV-2 surface-located envelope spike, which leads to prolonged activation of the alternative pathway $[1,2]$. 
Table 3. Laboratory parameters in case 3

\begin{tabular}{|c|c|c|c|c|c|c|}
\hline Parameters & $\begin{array}{l}\text { Normal } \\
\text { value }\end{array}$ & $\begin{array}{l}\text { At admission } \\
\text { (COVID-19) }\end{array}$ & $\begin{array}{l}\text { 11th day } \\
\text { (COVID-19) }\end{array}$ & $\begin{array}{l}\text { 18th day } \\
\text { eculizumab } \downarrow\end{array}$ & 22nd day & $\begin{array}{l}\text { 24th day } \\
\text { (death) }\end{array}$ \\
\hline $\mathrm{BP}(\max ), \mathrm{mm} \mathrm{Hg}$ & & $130 / 90$ & $140 / 90$ & $130 / 80$ & $110 / 80$ & $130 / 80$ \\
\hline Oxygen saturation, \% & $>96$ & 80 & $94-100$ & $94-100$ & $97-100$ & $95-98$ \\
\hline Hemoglobin, g/L & $120-150$ & 113 & 89 & 74 & 112 & 100 \\
\hline Thrombocytes $\times 10^{9} / \mathrm{L}$ & $180-320$ & 179 & 85 & 6 & 190 & 124 \\
\hline $\mathrm{LDH}, \mathrm{U} / \mathrm{L}$ & $<247$ & 503 & 1,900 & 2,860 & 600 & 812 \\
\hline Creatinine, $\mu \mathrm{mol} / \mathrm{L}$ & $49-90$ & 70 & $\begin{array}{l}193 \\
\text { Oliguria }\end{array}$ & 200 & 80 & 77 \\
\hline International normalized ratio & $0.85-1.2$ & 1.2 & 1.35 & - & 1.08 & - \\
\hline PT, s & $11-16$ & 14.4 & 15.9 & - & 12.5 & - \\
\hline aPTT, s & $21.1-36.5$ & 24.6 & 41.4 & - & 32.6 & - \\
\hline D-dimer, ng/mL FEU & $<500$ & 2,700 & 3,900 & 2,000 & 1,200 & 800 \\
\hline Proteinuria, g/g Cr & $<0.15$ & 1.0 & $\mathrm{n} / \mathrm{a}$ & 1.0 & $\mathrm{n} / \mathrm{a}$ & 0.5 \\
\hline Hematuria, in FOV & $0-1$ & $1-2$ & $\mathrm{n} / \mathrm{a}$ & $1-2$ & $\mathrm{n} / \mathrm{a}$ & $1-2$ \\
\hline
\end{tabular}

$\mathrm{LDH}$, lactate dehydrogenase; BP, blood pressure.

Magro et al. [3] described pulmonary and skin microvascular C5b-9 deposition in COVID-19 patients. Diao et al. [6] described renal C5b-9 deposition in patients with SARS-CoV-2, which strongly suggests kidney complement activation.

For now, there are only a few cases that described severe TMA development in COVID-19 patients. For example, the first case describes aHUS recurrence triggered by COVID-19 in a 28-year-old female [5], and the second case describes a child with COVID-19 who developed renal failure due to TMA [7]. Two patients who had COVID-19-associated TMA were presented in a case series study that focused on analyzing kidney biopsy results. One of the patients had a history of gemcitabine use [5].

However, we suggest that some cases of TMA can be missed. In a short time, we observed 3 cases of TMA in COVID-19 patients which required renal replacement therapy. It is not clear whether SARS-CoV-2 played a role in widespread endothelial damage through direct complement activation. Perhaps, it happened due to the genetic background. Unfortunately, in our study, genetic testing was not performed. It was shown that secondary complement-mediated TMA in COVID-19 can be associated with negative genetic tests for aHUS [7].

Eculizumab, a monoclonal antibody that binds C5 and blocks its cleavage, is the current reference standard treatment for aHUS and all cases with lab signs of complement activation, microangiopathic hemolysis, and kidney injury [8]. The duration of therapy varies depending on the patients' condition (stabilization and normalization of lab parameters and kidney function) as well as the results of genetic testing [9]. The use of anticomplement therapies such as eculizumab should be considered in refractory cases of progressive COVID-19 flow with TMA. Administration of anti-C5 monoclonal antibody eculizumab in 3 critical COVID-19 patients with suppressed total hemolytic complement levels and AKI led to a normalization of creatinine levels in 2 patients. The severe cases of complement activation seen in COVID-19 patients, as opposed to other cases of aHUS types of TMA, may require much higher doses of eculizumab than recommended [10].

The third case described a patient with severe COVID-19 with pneumonia that led to the development of acute TMA with microangiopathic hemolysis (progressive decrease of hemoglobin and a 10-fold increase of LDH levels), severe thrombocytopenia, and kidney damage. The onset of simultaneous TMA along with the secondary bacterial infection in a patient with COVID-19 gave us a reason to suspect DIC. It is very difficult to make a diagnosis of an aHUS or TTP type of TMA in the setting of active DIC [11]. The mechanism of DIC is the activation and consumption of coagulation factors followed by the secondary fibrinolysis leading to the elevation of the D-dimer level in the blood [12]. However, a significantly elevated D-dimer level in COVID-19 may also be due to severe inflammation. Despite the taken measures that included plasma therapy, 
we still noted elevated BP, hemolysis, thrombocytopenia, along with a decrease in the level of inflammatory markers and no signs of coagulation deterioration. In our case, normal levels of INR, PT, and PTT allowed us to exclude active DIC and suspected secondary hemolytic uremic syndrome triggered by COVID-19. After the complement-blocking therapy, the patient's hemoglobin level, thrombocytes count, as well as renal function stabilized. This allowed us to confirm the diagnosis. However, a secondary bacterial infection that complicated the COVID-19 flow made us interrupt the eculizumab treatment.

In summary, we observed 3 cases of TMA triggered by SARS-CoV-2. In the absence of other causes of TMA, we believe that aHUS is triggered by abnormal complement activation in COVID-19 patients. Eculizumab should be considered to treat these patients.

\section{Statement of Ethics}

Written informed consent was obtained from the patients and next of kin for publication of this case reports.

\section{Conflict of Interest Statement}

The authors have no conflicts of interest to declare.

\section{Funding Sources}

The authors did not receive any funding for this study.

\section{Author Contributions}

K.J. contributed to the acquisition, analysis, and interpretation of data of the study; C.N. contributed to the conception and design of the study, analysis, and interpretation of data for the study; A.E. contributed to the acquisition and interpretation of data of the study, S.Y. contributed to the acquisition and analysis of the study; S.E. and V.M. contributed to analysis and interpretation of data of the study; M.S. contributed to drafting the manuscript.

\section{Data Availability Statement}

All data generated or analyzed during this study are included in this article. Further inquiries can be directed to the corresponding author.

\section{References}

1 Zhou Y, Lu K, Pfefferle S, Bertram S, Glowacka I, Drosten C, et al. A single asparaginelinked glycosylation site of the severe acute respiratory syndrome coronavirus spike glycoprotein facilitates inhibition by mannosebinding lectin through multiple mechanisms. J Virol. 2010;84:8753-64.

2 Walls AC, Park YJ, Tortorici MA, Wall A, McGuire AT, Veesler D. Structure, function, and antigenicity of the SARS-CoV-2 spike glycoprotein. Cell. 2020;181:281-92. e6.

3 Magro C, Mulvey JJ, Berlin D, Nuovo G, Salvatore S, Harp J, et al. Complement associated microvascular injury and thrombosis in the pathogenesis of severe COVID-19 infection: a report of five cases. Transl Res. 2020;220: $1-13$.
4 Ville S, Le Bot S, Chapelet-Debout A, Blancho G, Fremeaux-Bacchi V, Deltombe C, et al. Atypical HUS relapse triggered by COVID-19. Kidney Int. 2021;99:267-9.

5 Sharma P, Uppal NN, Wanchoo R, Shah HH, Yang Y, Parikh R, et al. COVID-19-associated kidney injury: a case series of kidney biopsy findings. J Am Soc Nephrol. 2020;31:194858.

6 Diao B, Wang C, Wang R, Feng Z, Zhang J, Yang $\mathrm{H}$, et al. Human kidney is a target for novel severe acute respiratory syndrome coronavirus 2 (SARS-CoV-2) infection. Nat Commun. 2021 May 4;12(1):2506.

7 Mahajan R, Lipton M, Broglie L, Jain NG, Uy NS. Eculizumab treatment for renal failure in a pediatric patient with COVID-19. J Nephrol. 2020 Sep 26;33:1373-6.
8 Trimarchi H, Coppo R. COVID-19 and acute kidney injury in pediatric subjects: is there a place for eculizumab treatment? J Nephrol. 2020;33:1119-20.

9 Loirat C, Frémeaux-Bacchi V. Atypical hemolytic uremic syndrome. Orphanet J Rare Dis. 2011;6:60

10 Laurence J, Mulvey JJ, Seshadri M, Racanelli A Harp J, Schenck EJ, et al. Anti-complement C5 therapy with eculizumab in three cases of critical COVID-19. Clin Immunol. 2020;219:108555.

11 Wada H, Matsumoto T, Suzuki K, Imai H, Katayama N, Ibaal T, et al. Differences and similarities between disseminated intravascular coagulation and thrombotic microangiopathy. Thromb J. 2018;16:14

12 Wada H. Disseminated intravascular coagulation. Clin Chim Acta. 2004;344(1-2):13-21. 